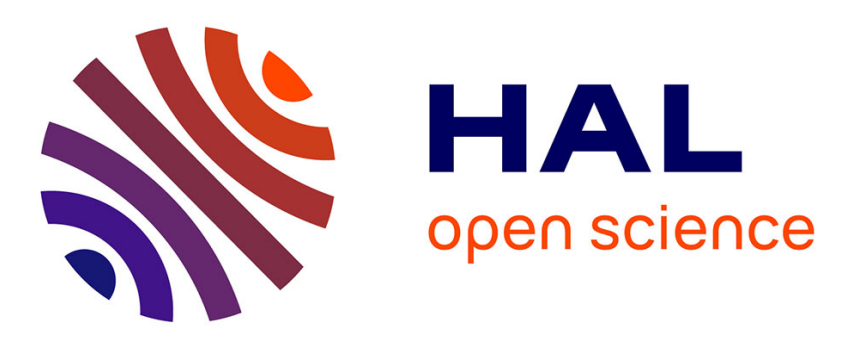

\title{
3D-printed adaptive acoustic lens as a disruptive technology for transcranial ultrasound therapy using single-element transducers
}

Guillaume Maimbourg, Alexandre Houdouin, Thomas Deffieux, Mickaël Tanter, Jean-François Aubry

\section{To cite this version:}

Guillaume Maimbourg, Alexandre Houdouin, Thomas Deffieux, Mickaël Tanter, Jean-François Aubry. 3D-printed adaptive acoustic lens as a disruptive technology for transcranial ultrasound therapy using single-element transducers. Physics in Medicine and Biology, 2018, 63 (2), pp.025026. 10.1088/13616560/aaa037. hal-02411212

\section{HAL Id: hal-02411212 \\ https://hal.science/hal-02411212}

Submitted on 14 Dec 2019

HAL is a multi-disciplinary open access archive for the deposit and dissemination of scientific research documents, whether they are published or not. The documents may come from teaching and research institutions in France or abroad, or from public or private research centers.
L'archive ouverte pluridisciplinaire HAL, est destinée au dépôt et à la diffusion de documents scientifiques de niveau recherche, publiés ou non, émanant des établissements d'enseignement et de recherche français ou étrangers, des laboratoires publics ou privés. 


\section{D-printed adaptive acoustic lens as a disruptive technology for transcranial ultrasound therapy using single-element transducers}

Guillaume Maimbourg ${ }^{1,2,3,4}$, Alexandre Houdouin ${ }^{1,2,3}$, Thomas Deffieux ${ }^{1,2,3}$, Mickael Tanter $^{1,2,3}$ and Jean-François Aubry ${ }^{1,2,3}$

1- INSERM U979, Institut Langevin, Paris, France;

2- ESPCI Paris, PSL Research University, Institut Langevin, Paris, France;

3- CNRS UMR 7587, Institut Langevin, Paris, France;

4- Université Paris Diderot, Paris, France

The development of multi-element arrays for better control of the shape of ultrasonic beams has opened the way for focusing through highly aberrating media, such as the human skull. As a result, the use of brain therapy with transcranial-focused ultrasound has rapidly grown. Although effective, such technology is expensive. We propose a disruptive, low-cost approach that consists of focusing a $1-\mathrm{MHz}$ ultrasound beam through a human skull with a single-element transducer coupled with a tailored silicone acoustic lens cast in a 3D-printed mold and designed using computed tomography-based numerical acoustic simulation. We demonstrate on $\mathrm{N}=3$ human skulls that adding lens-based aberration correction to a single-element transducer increases the deposited energy on the target 10 fold.

\section{Introduction}

In the 1950s, Fry et al. conducted pioneering experiments to evaluate the effects of High Intensity Focused Ultrasound (HIFU) on the Central Nervous System (CNS) [1, 2]. Nevertheless, ultrasound transcranial focusing remained out of reach for a long time due to the strong aberrating and attenuating properties of the skull $[3,4]$ that induce a significant defocusing of the ultrasonic beam. Fry's experiments had thus been conducted after craniotomy, resulting in an invasive procedure that limited the competitive interest of ultrasound compared to conventional brain surgery [5-7]. It was not until the 1990s that the possibility of actively shaping the wavefront to correct bone-induced aberrations emerged through the use of a multi-element transducer array whose phase could be adjusted individually [8-11]. Originally, this technique was minimally invasive, as it first required to position a reference transducer or hydrophone inside the brain, at the target location, to assess the phase profile $[9,12]$. It then became completely non-invasive, when it was demonstrated that accurate phase profiles could be obtained by numerical simulation $[10,13]$. This second method relies on a preliminary determination of the acoustic properties (density and sound velocity) of the skull, which are modeled from Computed Tomography (CT) [13, 14] or Magnetic Resonance (MR) [15] acquisition. An acoustic 
wave is then emitted virtually from the target and propagates through the skull, up to the multi-element transducer. The arrival times are recorded at the surface of the transducer. The phase correction to be applied on each element of the array is then calculated by time reversal $[8,9]$ or phase shift $[10,11]$. The quality of such focusing in terms of acoustic intensity in the brain or location of the maximum intensity has been demonstrated on animals [16-18] and human cadavers [19-21]. Such procedure allowed for the development of new therapeutic methods for non-invasive transcranial brain therapy like blood-brain-barrier (BBB) opening [22-25], thermal lesioning by HIFU $[17,18,26]$ and neurostimulation/neuromodulation [27-32] up to clinical trials [33-36]. Thanks to the progress made in electronics, especially in transducer technology, therapy devices have been designed with an exponentially growing number of elements so as to improve the focusing quality: 64 elements in 2000 [37], 300 in 2003 [38] and 1024 in 2012 [39].

In this paper, we propose a complete rupture with this approach by performing adaptive transcranial focusing with a single-element focused transducer combined with a patient-specific, tailor-made silicone acoustic lens of controlled thickness. Acoustic lenses have been successfully used in the past to create multiple foci or enlarge treated volume [40-47] and to target subcutaneous areas with high $f$ numbers [48]. Capitalizing on the recent progress of 3D printing, we describe here how to focus a high-frequency $(914 \mathrm{kHz})$ ultrasonic beam through the human skull, by the combination of a single-element transducer and an acoustic lens specifically designed for each one of the skulls used in the study. The objective is to experimentally validate the relevance of a single-element transducer combined with an acoustic lens to focus ultrasound in the CNS through the skull.

\section{Methods}

\section{Transducer}

A single-element transducer with a $59 \mathrm{~mm}$ radius of curvature and a $67 \mathrm{~mm}$ aperture (H101 MR, Sonic Concepts, Bothell, USA) was used to achieve transcranial focusing. It was operated at one of its resonant frequencies, $914 \mathrm{kHz}$, which were determined from the analysis of its complex impedance against frequency, with a vector network analyzer (ZVL, Rohde \& Schwarz, Munich, Germany).

\section{Human skulls}

The experiments were conducted on $\mathrm{N}=3$ human skulls (hereafter referenced as $\mathrm{A}$, $B$ and $C$ ). The human skulls were provided by the Institut d'Anatomie (UFR Biomédicale des Saints-Pères, Université Paris Descartes, Paris, France) and tattooed with individual numbers, as approved by the ethics committee of the Centre du Don des Corps (Université Paris Descartes, Paris, France). Before CT-scans and sonications, the skulls were immersed in water and degassed under a $2 \mathrm{mbar}$ reduced pressure (diaphragm pump FB65457, Fischer Scientific, Waltham, MA, USA) during 48h; their CT-scans were acquired (Sensation 64, Siemens, Erlangen, 
Germany) at the $\mathrm{CHU}$ de Marseille Nord, France. The in-plane spatial resolution of the slices was $0.41 \mathrm{~mm}$, while slice thickness and interslice spacing were $0.60 \mathrm{~mm}$ and $0.30 \mathrm{~mm}$, respectively. Voxels displaying values beneath 0 Hounsfield Units (HU) were set to $H U_{\min }=0$, which is that of water. Voxels with values above 2400 were set to $H U_{\max }=2400$, the expected value for cortical bone [49]. We used a linear relationship between the HU from the CT-scans, sound speed, and density [50]. At $21^{\circ} \mathrm{C}$, the speed of sound in water equals $c_{\text {water }}=1485 \mathrm{~m} / \mathrm{s}$ [51]. We chose the following parameters for the cortical bone: $c_{\text {bone }}=3100 \mathrm{~m} / \mathrm{s}$ and $\rho_{\text {bone }}=1900 \mathrm{~kg} / \mathrm{m}^{3}$ [51]. Speed and density in the medium are then derived by a linear approximation [49]:

$$
\begin{aligned}
c(x, y, z) & =c_{\text {water }}+\left(c_{\text {bone }}-c_{\text {water }}\right) \times \frac{H U(x, y, z)-H U_{\min }}{H U_{\max }-H U_{\min }} \\
\text { and } \quad & \rho(x, y, z)=\rho_{\text {water }}+\left(\rho_{\text {bone }}-\rho_{\text {water }}\right) \times \frac{H U(x, y, z)-H U_{\min }}{H U_{\max }-H U_{\min }}
\end{aligned}
$$

\section{Silicone used for lens material}

The acoustic lens fabricated in this study is made from commercial silicone (Elite double 8, Zhermack, Badina Polesine, Italy). As its physical properties were not provided by the manufacturer, we first measured its speed of sound and density, which are required to compute the thickness profile of the lens. A cylindrical sample of the silicone was made with a thickness $e=25.2 \pm 0.5 \mathrm{~mm}$, The density $\rho_{\text {lens }}$ of the material was evaluated by measuring the mass and volume of the sample. We obtained $\rho_{\text {lens }}=1.04 \pm 0.10 \mathrm{~g} . \mathrm{cm}^{-3}$. Then, we measured the speed of sound in the silicone with a $3 \mathrm{MHz}$ broadband ultrasonic transmitter-receiver pair (model 358, Imasonic, Besançon, France). The emitter was excited by a 3-cycle sinusoidal burst at $914 \mathrm{kHz}$ (AFG3101C, Tektronix, Beaverton, OR, USA). The signal was recorded with an oscilloscope (DPO3034, Tektronix, Beaverton, OR, USA) at a distance of $2 \mathrm{~cm}$ from the transmitter. The experiment was conducted at $21^{\circ} \mathrm{C}$, the reference temperature for all the experiments throughout the study. The time of flight of the signal in water $t_{\text {water }}$ was first evaluated. The sample $\gamma$ was then placed between the transducers so as to measure the time of flight $t_{\text {sample. }}$. Using $c_{\text {water }}=1485 \mathrm{~m} / \mathrm{s}$, the speed of sound in silicone $c_{\text {lens }}$ is given by:

$$
c_{\text {lens }}=\frac{c_{\text {water }}}{1+\frac{c_{\text {water }}\left(t_{\text {sample }}-t_{\text {water }}\right)}{e}}
$$

The speed of sound in the silicone was assessed at $914 \mathrm{kHz}$. The variation in duration for each case, with and without the silicone sample, was given by the relation:

$$
t_{\text {sample }}-t_{\text {water }}=8.2 \pm 0.5 \mu \mathrm{s}
$$

The speed of sound in the silicone can thus be figured out according to formula (3): 


$$
c_{\text {lens }}=1000 \pm 20 \mathrm{~m} / \mathrm{s}
$$

\section{Numerical simulation for acoustic lens thickness determination}

The corrective acoustic lens thickness was derived from the phase profile obtained by numerical propagation of an acoustic wave across the skull, and emitted from the target point. The simulated emitter was placed at a known location relative to the skull so as to target a predefined region (Figure 1.A1). The acoustic maps for the simulation were constructed from the speed of sound and density maps of the skull. A 3-dimension linear interpolation of the maps was performed to obtain a simulation grid, with a $\lambda_{\text {water }} / 10$ spatial step. The time step $\Delta t_{\text {sim }}$ was computed based on a 0.3 Courant-Friedrichs-Lewy (CFL) condition in the cortical bone. The target was used as a point source for the simulation. The number of time steps $N_{\text {sim }}$ was chosen so that the forward wave reaches the transducer. The simulation was conducted using a k-space pseudospectral method-based solver, k-Wave [52]. Absorption of the skull or brain tissue is not taken into account. To further reduce the simulation time, the point source at the target was replaced with an equivalent spherical wavefront originated from the target and adjusted as close as possible to the brain-skull interface. Figure 2 shows a cross-section view of the numerical simulation setup. A 2-cycle sinusoidal burst with Gaussian apodization was emitted at the frequency $f_{0}^{s i m}=914 \mathrm{kHz}$ and numerically propagated in the medium (Figure 1.A2). The signal $\tilde{s}\left(M, 1 \cdots N_{\text {sim }}\right)$ was recorded over the surface of the transducer, discretized by points generically named $M$ (Figure 1.A3). The phase $\varphi$ of the signal was evaluated by a projection onto the Fourier basis. The phase on each point $M$ on the surface of the transducer is thus given by:

$\varphi(M)=\arg \left(\sum_{n=1}^{n=N_{\text {sim }}} \tilde{s}(M, n) \times\left[\cos \left(2 \pi f_{0}^{\text {sim }} n \Delta t_{\text {sim }}\right)+i \sin \left(2 \pi f_{0}^{\text {sim }} n \Delta t_{\text {sim }}\right)\right] \Delta t_{\text {sim }}\right)$ 


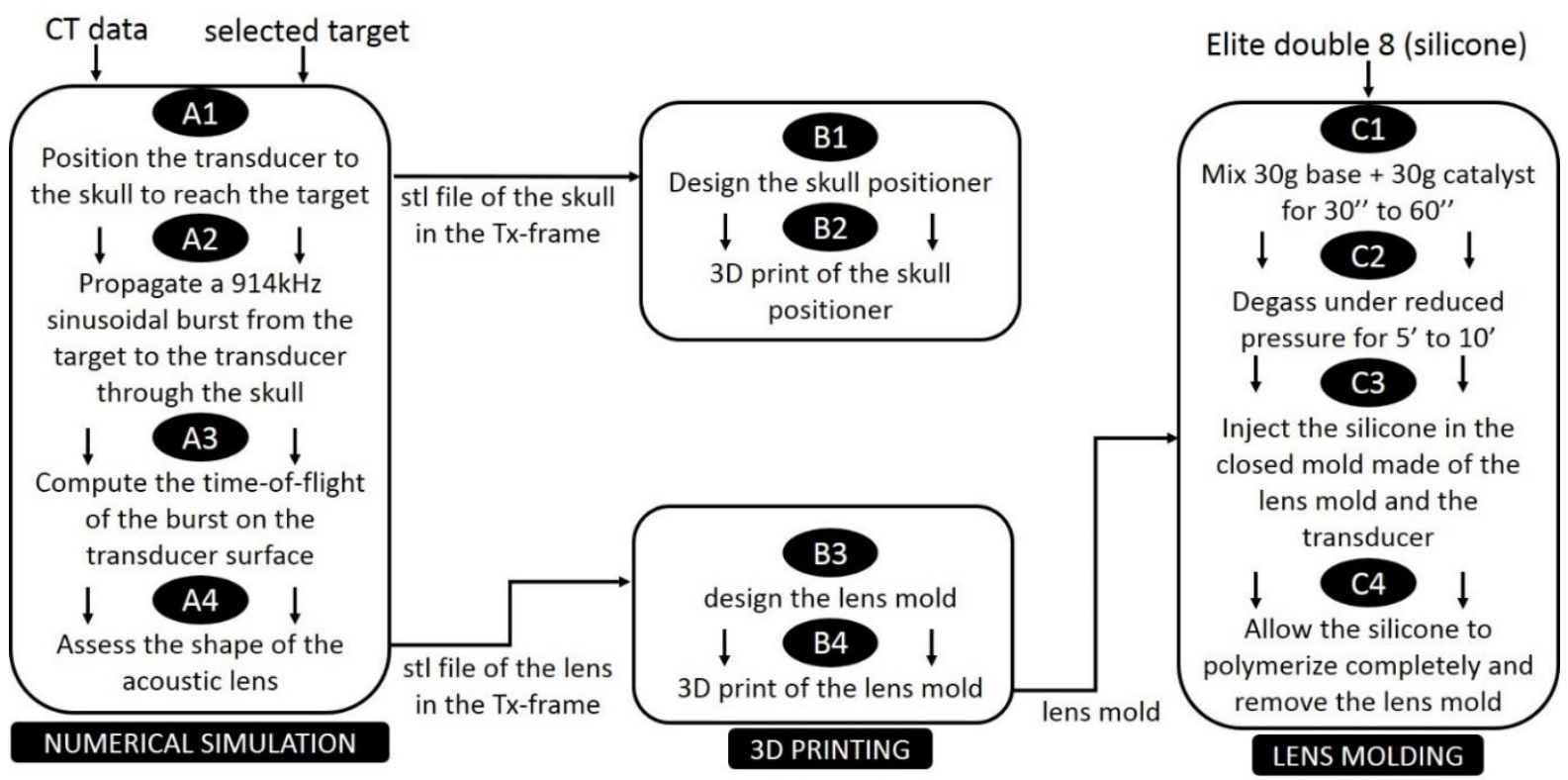

Figure 1: Synoptic view of the successive operations for the construction of the acoustic lens that corrects the aberrations of a skull, based on its CT. There are three specific steps to be distinguished: the numerical simulation, which gives us the desired thickness profile for the acoustic lens; the 3D printing of the skull-specific pieces (transducer-positioner and lens mold); lastly, the fabrication of the silicone acoustic lens using the aforementioned lens mold.

The phase was then 2D-unwrapped with the Matlab toolbox "2D phase unwrapping algorithms" based on the Goldstein's branch cut method [53] to avoid phase jumps. We have indeed noticed, during preliminary tests, that such phase jumps induced thickness anomalies which were detrimental to the focusing by causing refraction and diffraction of the beam. The local thickness $p$ of the lens on every point $M$ of the transducer is evaluated (Figure 1.A4), according to the following formula, and where $\tilde{\varphi}$ is the phase after unwrapping:

$$
p(M)=\frac{\widetilde{\varphi}(\mathrm{M})}{2 \pi f_{0}^{\text {sim }}} \cdot \frac{1}{1 / c_{\text {water }}-1 / c_{\text {lens }}}+K
$$

$K$ is a constant length chosen to set the minimal thickness of the lens. In our study, $K$ was adjusted to $2 \mathrm{~mm}$. Refraction was not taken into account. We assume that refraction plays a limited role because the acoustic lens has a spherical profile, which matches that of the wavefront. The angle of incidence is thus close to zero. 


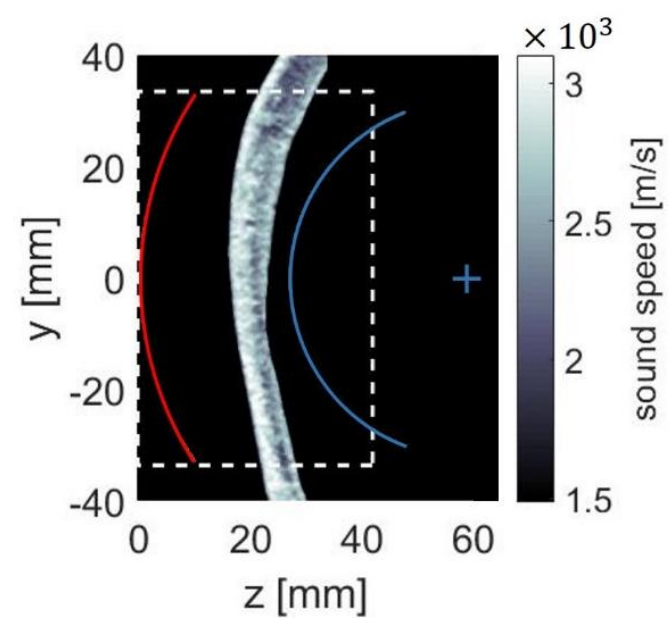

Figure 2: Speed of sound map of the skull $A$ in a cross-section plane defined by $x=0$. The blue cross represents the target and coincides with the geometrical focus of the transducer. The blue arc is the source using in the simulation, centered on the target. The red surface

represents the transducer surface where the acoustic signal is recorded after it has propagated through the skull. We obtain the phase profile on the surface of the transducer.

The white dash box delimits the area in which the simulation was running.

The simulation was run on a workstation equipped with two 2.20GHz Intel Xeon CPU E5-2630v4 processors and 32GB RAM; the full running time was about one hour.

\section{Assessing the efficiency of the lens-based aberration correction through numerical simulations.}

The lens thickness is directly calculated from the difference in speed of sound between the lens material and water (Eq. 5). Given that refraction and diffraction phenomena could impact the quality of the focusing, we simulated the propagation for five configurations, in an attempt to better evaluate their influence:

1) Without the skull; this simulates the shape of the focal spot in water only.

2) With a skull but no correction applied to it.

3) With a skull on which a correction is applied. This correction is made using an array featuring as many elements as the number of points from which the lens profile was computed. Each emitting voxel acts as a transducer with a $\frac{\lambda_{\text {water }}}{10} \times \frac{\lambda_{\text {water }}}{10}$ surface. Such was our theoretical gold standard that is impossible to achieve in practice.

4) With a skull corrected by an array consisting of $6 \times 6 \mathrm{~mm}^{2}$ elements, which corresponds to typical transcranial setups with small multi-element transducers [50]. This configuration corresponds to the realistic gold standard.

5) With a skull corrected by the acoustic lens.

These tests were conducted on skull A only.

\section{Casting of the tailor-made lens}

Using the thickness profile of the lens that we obtained through numerical simulation, a casting mold was 3D-printed (Replicator 2X, Makerbot, Brooklyn, NY, USA) with a 
$300 \mu \mathrm{m} X Y$ resolution and a $100 \mu \mathrm{m} \mathrm{Z}$ resolution (Figure 1.B3-4). A photography of the mold is shown in Figure 3.B. This mold was then fastened to the transducer with 6 screws (Figure 3.A). The silicone was prepared, according to user instructions, by mixing the catalyst with the base (1:1 ratio). The blend was then degassed under reduced pressure $(<2 \mathrm{mbar})$ for 10 minutes. Once the pouring was complete, the lens polymerized for 20 minutes (Figure 1.C1-4). The mold was then removed, leaving the silicone lens in direct contact with the transducer. A visual check was carried out to ensure the absence of air bubbles that could have been trapped during the pouring stage.
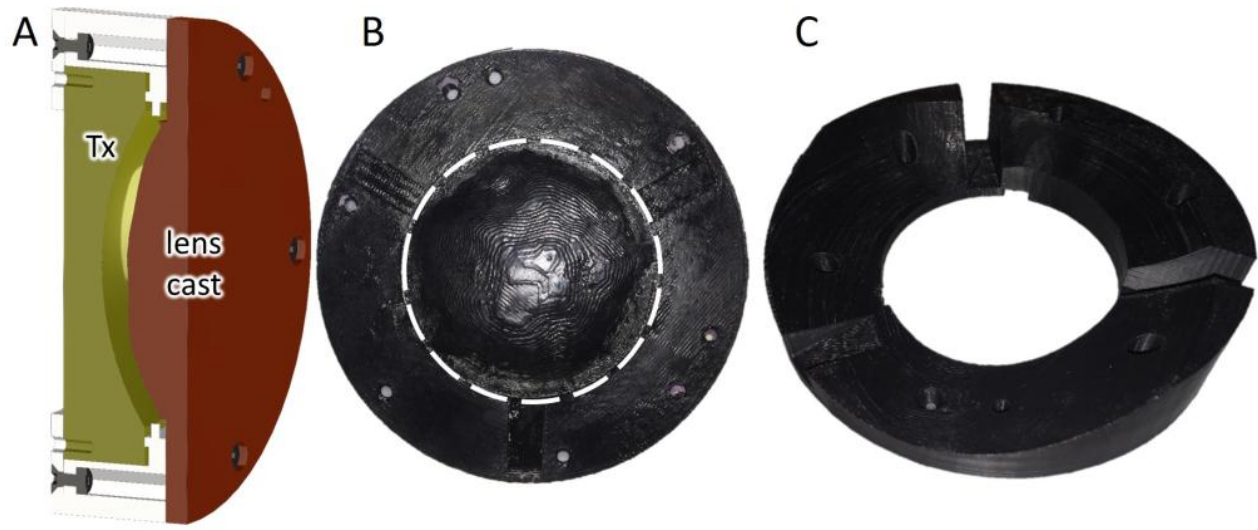

Figure 3: (A) axial cross-section view of the setup used for casting the acoustic lens. The free space between the transducer $(T X)$ and the lens mold is filled with silicone to shape the lens. (B) 3D-printed mold of the acoustic lens used to shape the lens (skull $A$ ). The silicone will be poured in the area marked by the white dashes. (C) 3D-printed skull holder created for skull $A$. Its surface is designed to conform to the shape of the skull.

\section{D-printed transducer positioning setup}

In this proof of concept study, we chose to position the transducer relative to the skull in a simple and effective manner, without a clinical stereotactic frame. To do so, a holder was tailor-made for each skull with a 3D-printer (Replicator 2X, Makerbot, Brooklyn, NY, USA). Taking into account the position of the transducer relatively to the skull, and the skull surface determined from the CT-scan, a plastic holder of complementary shape to the skull was fabricated and attached to the transducer (Figure 4.A-B); the holder presents a surface that matches the imprint of the skull (Figure 3.C).

Two rubber bands were used to maintain the transducer on the skull throughout the experiments (Figure 4.B). 

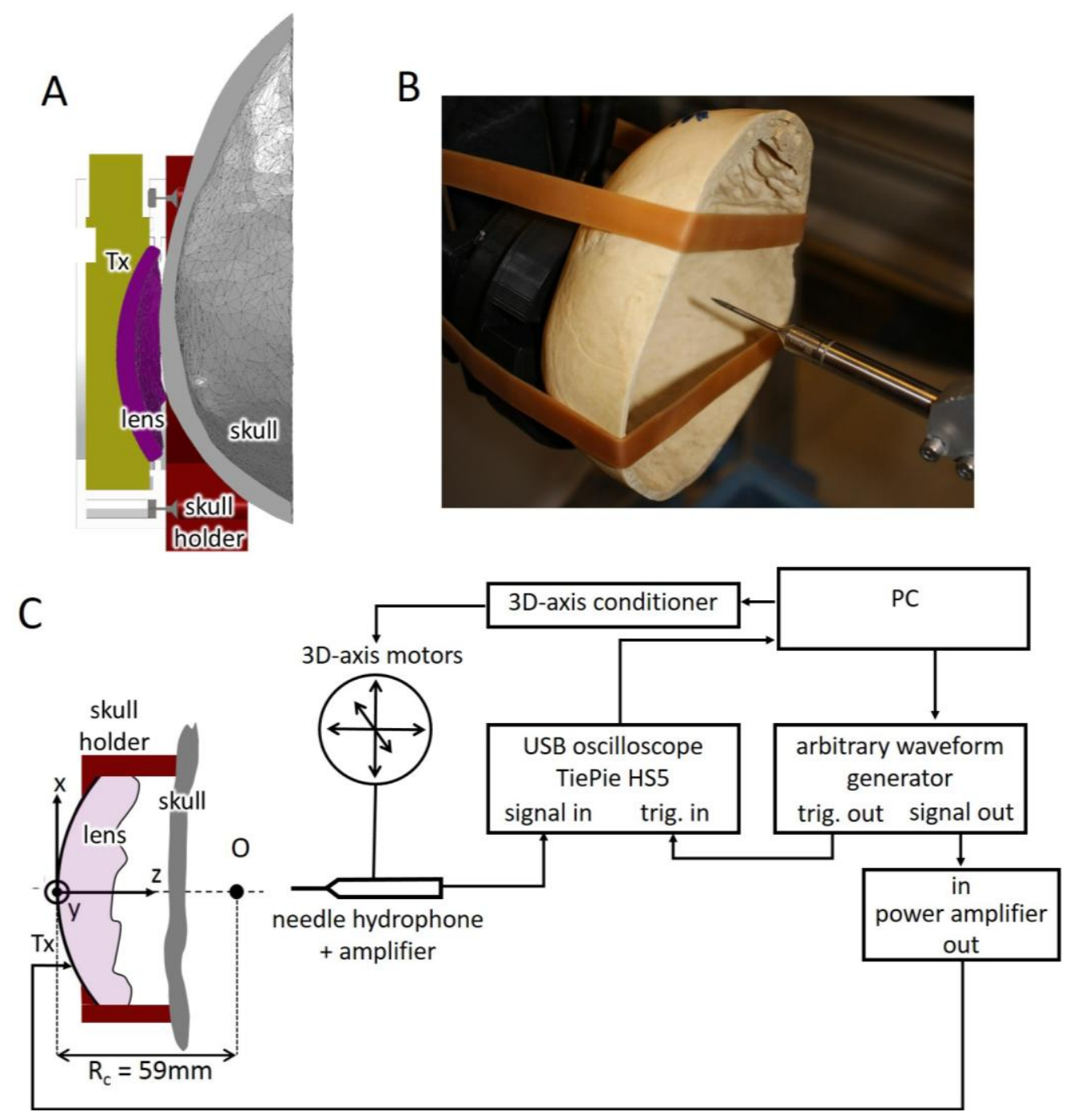

Figure 4: (A) Cross-section view of the experimental setup. The acoustic lens is in direct contact with the transducer. The holder ensures the positioning of the transducer and lens, relatively to the skull. (B) In situ photography depicting the acquisition of a pressure field behind a skull. The skull is held in contact with the holder with two rubber bands. (C) Experimental setup used to evaluate the efficiency of the acoustic field correction done by the lens. The transducer was powered by a signal provided by an arbitrary waveform generator and amplified. The pressure field was measured using a needle hydrophone mounted on a 3-axis positioning system. The acoustic signal was then amplified by a low noise amplifier before digitalization. Monitoring is done using a PC. Depending on the situation, the skull and/or the acoustic lens are positioned in front of the transducer. The Cartesian coordinate system used for the purposes of the study is also shown. The axes correspond to the main axes of the 3-axis positioning system of the hydrophone. The z-axis coincides with the longitudinal axis of the transducer. The geometric focus of the transducer coincides with the origin $O$ of the coordinate system.

\section{Acoustic field measurements}

The acoustic lens was fabricated as previously explained, and the skull was positioned relatively to the transducer using the holder. A cross-section view of this 
setup is shown in Figure 4.A. An arbitrary waveform generator (AFG3101C, Tektronix, Beaverton, OR, USA) emitted a 200 -cycle sinusoidal burst at $914 \mathrm{kHz}$. This signal was then amplified (40AD1, Amplifier Research, Souderton, PA, USA). An oscilloscope (DPO3034, Tektronix, Beaverton, OR, USA) monitored the input and output signals of the amplifier. The acoustic pressure field was measured with a needle hydrophone (HNA-0400, Onda Corp., Sunnyvale, CA, USA) mounted on a 3axis positioning system (ESP301 and ILS Linear Stages, Newport, CA, USA). The $x$, $y$ and $z$ axes corresponded to the translational directions of the scan, so that $z$ corresponded to the longitudinal axis of the transducer (Figure 4.C). The output signal of the hydrophone was amplified (AG-2020 and AH-2020-DCBSW, Onda Corp., Sunnyvale, CA, USA) before digitalization at the sampling frequency $f_{s}=$ $100 \mathrm{MHz}$ on $N_{\text {exp }}=1 \mathrm{MPts}$ using a data acquisition card (HS5, TiePie, Sneek, Netherlands). All the devices were synchronized using a computer, thanks to an inhouse Matlab code (Mathworks, Natick, MA, USA). The synchronized acquisition code was adapted from the template codes provided by the TiePie company [54]. A schematic overview of the experimental setup is shown in Figure 4.C. For each position $P$ of the hydrophone, the signal was averaged throughout 5 acquisitions. In order to extract the harmonic component $A_{m}$ from the acoustic signal, the average signal $\tilde{s}$ was projected onto the Fourier basis corresponding to the excitation frequency $f_{0}^{\text {exp }}=914 \mathrm{kHz}$. That is to say, with $\Delta t=1 / f_{s}$ :

$$
A_{m}(P)=\left|\sum_{n=1}^{n=N_{s i m}} \tilde{S}(P, n) \times\left[\cos \left(2 \pi f_{0}^{\text {exp }} n \Delta t\right)+i \sin \left(2 \pi f_{0}^{\text {exp }} n \Delta t\right)\right] \Delta t\right|
$$

\section{Experimental protocol for each skull}

Skull-induced attenuation

The acoustic attenuation induced by each of the three skulls was assessed. To this end, a $30 \mathrm{~mm}$ radius circular scan $\Lambda$ was done in a transverse plane (center of the scan at $x=0 \mathrm{~mm}, y=0 \mathrm{~mm}$ and $z=-10 \mathrm{~mm}$, resolution $\Delta x=\Delta y=$ $0.5 \mathrm{~mm}$ ) with and without the skulls. The total acoustic intensity $I_{\text {tot }}$ on the surface $\Lambda$ was calculated as follow:

$$
I_{\text {tot }}=\sum_{\text {surface }} \frac{A_{m}^{2} \Delta x \Delta y}{2 Z}
$$

where $Z=1.485$ MRay is the acoustic impedance of water. The attenuation $\alpha_{\text {skull }}$ induced by the skull (in $\mathrm{dB}$ ) is thus calculated.

$$
\alpha_{\text {skull }}=10 \log _{10}\left(\frac{I_{\text {tot }}(\text { with skull })}{I_{\text {tot }}(\text { without skull })}\right)
$$

Lens-induced attenuation

The lens-induced attenuation was also measured. To this end, an identical $30 \mathrm{~mm}$ 
radius circular scan $\Lambda$ was performed without lens, and then with the lens corresponding to each skull ( $A, B$ and $C$ ).

The lens-induced attenuation $\alpha$ (in $\mathrm{dB}$ ) is given by:

$$
\alpha_{\text {lens }}=10 \log _{10}\left(\frac{I_{\text {tot }}(\text { with lens })}{I_{\text {tot }}(\text { without lens })}\right)
$$

\section{Assessing the efficiency of the acoustic lens correction}

The quality of the focusing through the skull, with and without the acoustic lens was assessed. To do so, three successive scans were conducted around the acoustic focus $F(0 \mathrm{~mm}, 0 \mathrm{~mm}, 0 \mathrm{~mm})$ : a reference scan in water, an aberrated scan through the skull and a lens-based corrected scan, as illustrated in Table 1. The pressure was recorded in an ellipsoidal volume with a $9 \mathrm{~mm}$ semi-major axis $(0.5 \mathrm{~mm}$ resolution) in the axial direction and an $8 \mathrm{~mm}$ semi-minor axis $(0.3 \mathrm{~mm}$ resolution) in the transverse directions.

\begin{tabular}{|c|c|c|c|}
\cline { 2 - 4 } \multicolumn{1}{c|}{} & reference scan & aberrated scan & corrected scan \\
\hline skull & & $\mathrm{X}$ & $\mathrm{X}$ \\
\hline acoustic lens & & & $\mathrm{X}$ \\
\hline & & & \\
& & & \\
& & & \\
\hline
\end{tabular}

Table 1: The configuration for each of the three scans. The reference scan is that of freespace propagation in water only; the aberrated scan is that of propagation across the skull; the corrected scan is that of propagation across both the lens and the skull.

\section{Data analysis}

Acoustic intensity $I_{\mathrm{ac}}$ was evaluated based on the pressure scans provided by the hydrophone. With $A_{m}$ being the pressure amplitude and $Z$ the impedance of water,

$$
I_{\mathrm{ac}}=\frac{A_{m}^{2}}{2 Z}
$$

Throughout the rest of the paper, the attenuation (in $\mathrm{dB}$ ) induced by the skull will be subtracted from the acoustic intensity of the reference scan (Table 2), meaning that the reference scan can be seen as the result of an optimal focusing through the 
skull. In a similar manner, the attenuation (in $\mathrm{dB}$ ) induced by the lens will be added to the acoustic intensity of the corrected scan. Comparisons are thus carried out with equal intensity on the outer surface of the skull.

In particular, we computed the intensity obtained at the acoustic focus. We also estimated the position of the acoustic focus by calculating the barycentric coordinates of the intensity within the $-6 \mathrm{~dB}$ main focal region.

$$
\overrightarrow{O F}=\left(\begin{array}{l}
x_{F} \\
y_{F} \\
z_{F}
\end{array}\right)=\frac{\sum_{\mathrm{V} @-6 \mathrm{~dB}} I_{\mathrm{ac}}(M) \overrightarrow{O M} \Delta x \Delta y \Delta z}{\sum_{\mathrm{V} @-6 \mathrm{~dB}} I_{\mathrm{ac}}(M) \Delta x \Delta y \Delta z}
$$

The longitudinal error $\varepsilon_{\text {longi }}$ and transverse error $\varepsilon_{\text {trans }}$ are then defined by:

$$
\varepsilon_{\text {trans }}=\sqrt{x_{F}{ }^{2}+y_{F}{ }^{2}} \text { and } \varepsilon_{\text {longi }}=\left|z_{F}\right|
$$

The volume of the $-3 \mathrm{~dB}$ focal region was computed to assess energy dispersion for the reference $\left(V_{\text {ref }}\right)$, the aberrated $\left(V_{\text {aber }}\right)$ and the corrected scans $\left(V_{\text {cor }}\right)$.

We then calculated the percentage of acoustic intensity within the $-3 \mathrm{~dB}$ focal spot in the target area $V_{\text {ref }}$ for both the aberrated and the corrected scans:

and

$$
\begin{aligned}
& I_{\text {aber }}(\%)=\left(\frac{\sum_{\text {vref }} \frac{A_{\mathrm{m}, a b e r}^{2} \Delta x \Delta y \Delta z}{2 Z}}{\sum_{\text {Vtot }} \frac{A_{\mathrm{m}, \mathrm{aber}}^{2} \Delta x \Delta y \Delta z}{2 Z}}\right) \times 100 \\
& I_{\text {cor }}(\%)=\left(\frac{\sum_{\text {vee }} \frac{A_{\mathrm{m}, \mathrm{cor}}^{2} \Delta x \Delta y \Delta z}{2 Z}}{\sum_{\text {vtot }} \frac{A_{\mathrm{m}, \mathrm{cor}} \Delta x \Delta y \Delta z}{2 Z}}\right) \times 100
\end{aligned}
$$

\section{Results}

\section{Numerical simulation for assessing the shape of the acoustic lens}

The shape of the lens, defined to correct skull-induced aberrations, was obtained through numerical simulation. The results of the simulation run for skull $A$ are displayed in Figure 5. Figure 5.A shows a color scale map of the lens thickness on the whole surface of the transducer; Figure 5.B shows a cross-section view of said lens. For skull A, lens thickness was comprised between $2.0 \mathrm{~mm}$ and $11.3 \mathrm{~mm}$. 

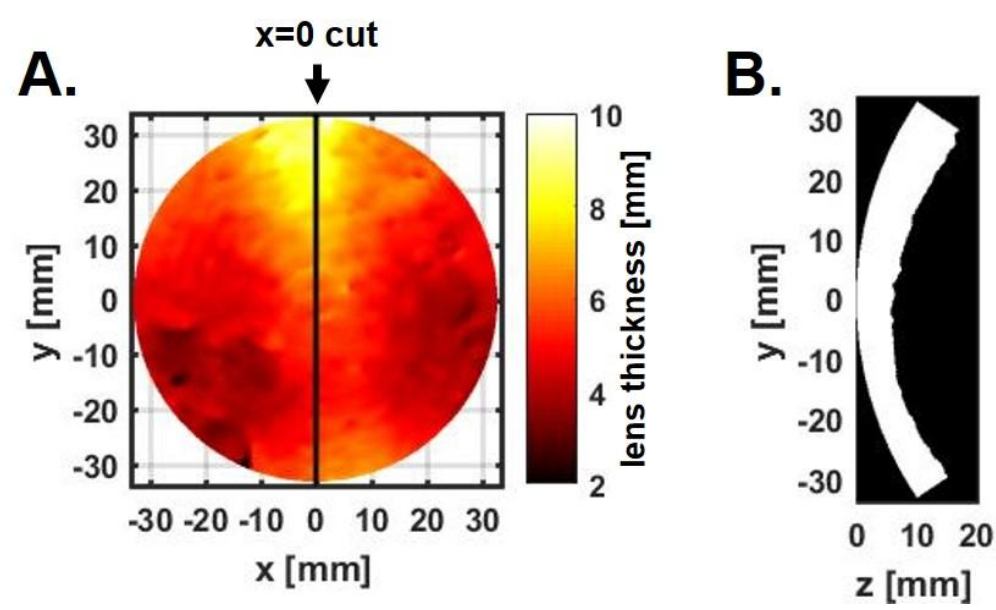

Figure 5: (A) Color-coded map of the thickness of the acoustic lens associated with skull $A$.

$(B)$ cross section view of the volumetric shape of the lens for the plane defined by $x=0$.

\section{Assessing the acoustic attenuation induced by the lens and the skull}

The first step was to assess the attenuation induced by the presence of the lens and the skull for each of the skulls tested (A, B, and $C)$. On average, skull acoustic attenuation equals $12.0 \pm 1.0 \mathrm{~dB}$ (Table 2) and lens-induced attenuation sits at $0.96 \pm 0.19 \mathrm{~dB}$.

skull $A$ skull $B$ skull $C$ mean \pm std

\begin{tabular}{|l|c|c|c|c|}
\hline skull & -11.5 & -13.1 & -11.3 & $12.0 \pm 1.0$ \\
\hline lens & -1.08 & -0.74 & -1.07 & $0.96 \pm 0.19$ \\
\hline
\end{tabular}

Table 2: Acoustic transmission loss for each passage across the skulls and acoustic lenses [in $d B$ ]

\section{Numerical simulation for assessing the efficacy of the lens-based aberration correction}

Figure 6 shows the results obtained through numerical simulations. Acoustic intensity is normalized by the maximum acoustic intensity obtained in case 2 (propagation through the skull without any correction). The $-3 \mathrm{~dB}$ isoline obtained for free-space focusing is given for comparison purposes. In all three cases where correction is applied, the acoustic intensity is concentrated in the immediate vicinity of the target, and the side lobes are significantly reduced in intensity. Furthermore, the intensity distribution profile matches the shape obtained in the free-space propagation case. The maximum intensity reached for each configuration is displayed on each 2D plot. The gold standard (correction using elements as small as the size of the spatial step of the simulation) provides a $+272 \%$ gain in acoustic intensity, compared to the no-correction case. The acoustic lens method increases the intensity at the focal spot by $+201 \%$. It therefore outperforms multi-element transducers featuring realistically-sized elements $\left(6 \times 6 \mathrm{~mm}^{2}\right)$, which provide a $+180 \%$ gain in intensity at the focal spot only. 

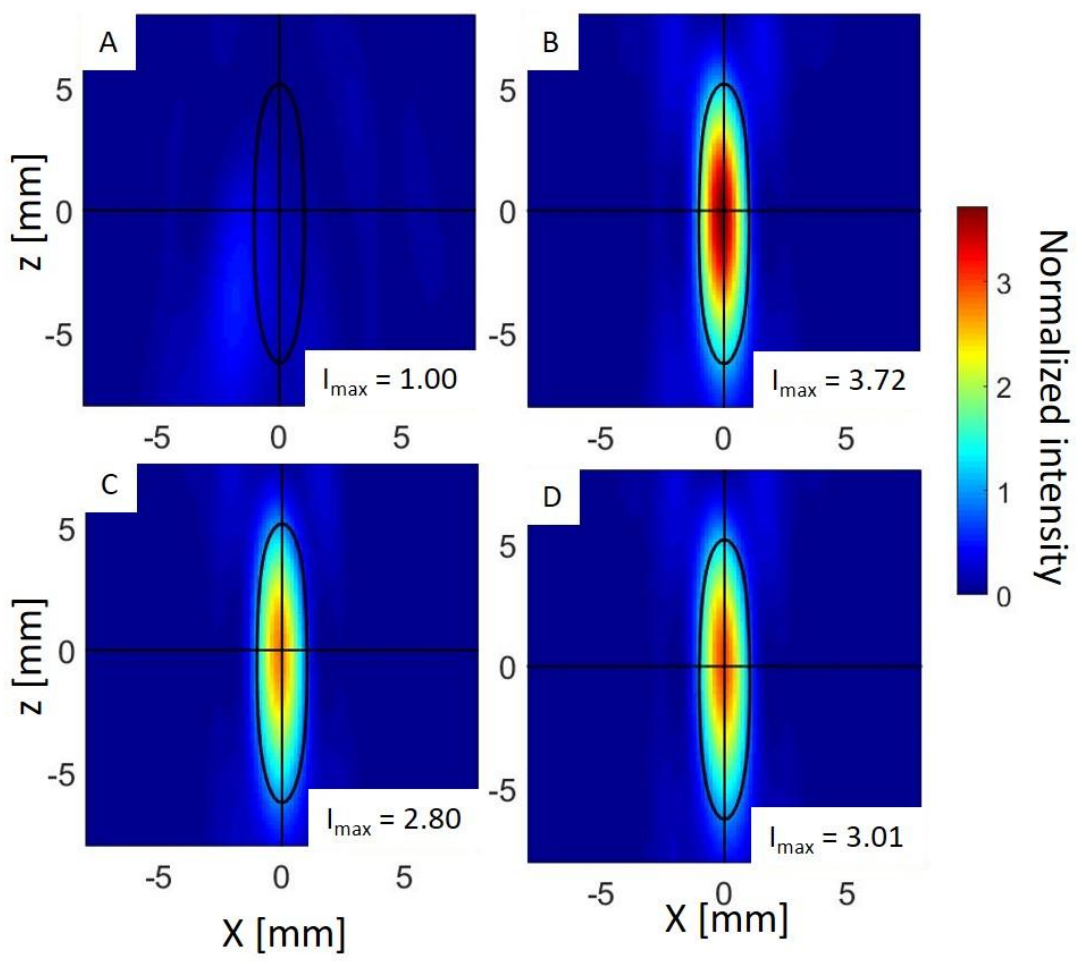

Figure 6: Longitudinal view of the acoustic intensity in the targeted area for skull A. A: aberrated; $B$ : correction using an array with as many elements as the number of points from which the lens profile was computed (theoretical gold standard); C: correction using an array of $6 \times 6 \mathrm{~mm}^{2}$ elements (realistic gold standard), D: correction using the acoustic lens. The black cursor is centered on the target, and the reference (free propagation, no skull) -3dB focus area is outlined in black (oval shape).

\section{Position errors of the focus}

The acoustic focus, without lens and skull, was taken as a reference. It was set as the origin of the coordinate system for the three scans, matching the target. In Figure 7 , an axial view $(\mathrm{z}=0)$ and a transverse view $(\mathrm{y}=0)$, centered on the acoustic focal, are presented for skull A without aberration correction (left), and with the lens-based aberration correction (right). The $-3 \mathrm{~dB}$ isoline computed for the reference scan is drawn (black bold line); the cursor represents the target. Intensities are normalized to unit (divided by the maximum reached throughout the volume) and expressed using a linear scale. Qualitatively speaking, we notice the presence of several foci on the aberrated scan, outside the target. By contrast, the lens-based correction produces a single focus spot centered in the immediate vicinity of the target. Quantitatively speaking, the longitudinal and transverse position errors from the target were evaluated and indexed in Table 3. On average, the correction offered by the lens reduces the transverse errors from $2.13 \mathrm{~mm}$ to $0.36 \mathrm{~m}$. For the sake of comparison, the Full Width at Half Maximum (FWHM) of the reference focus is $1.5 \mathrm{~mm}$ in the transverse plane. 


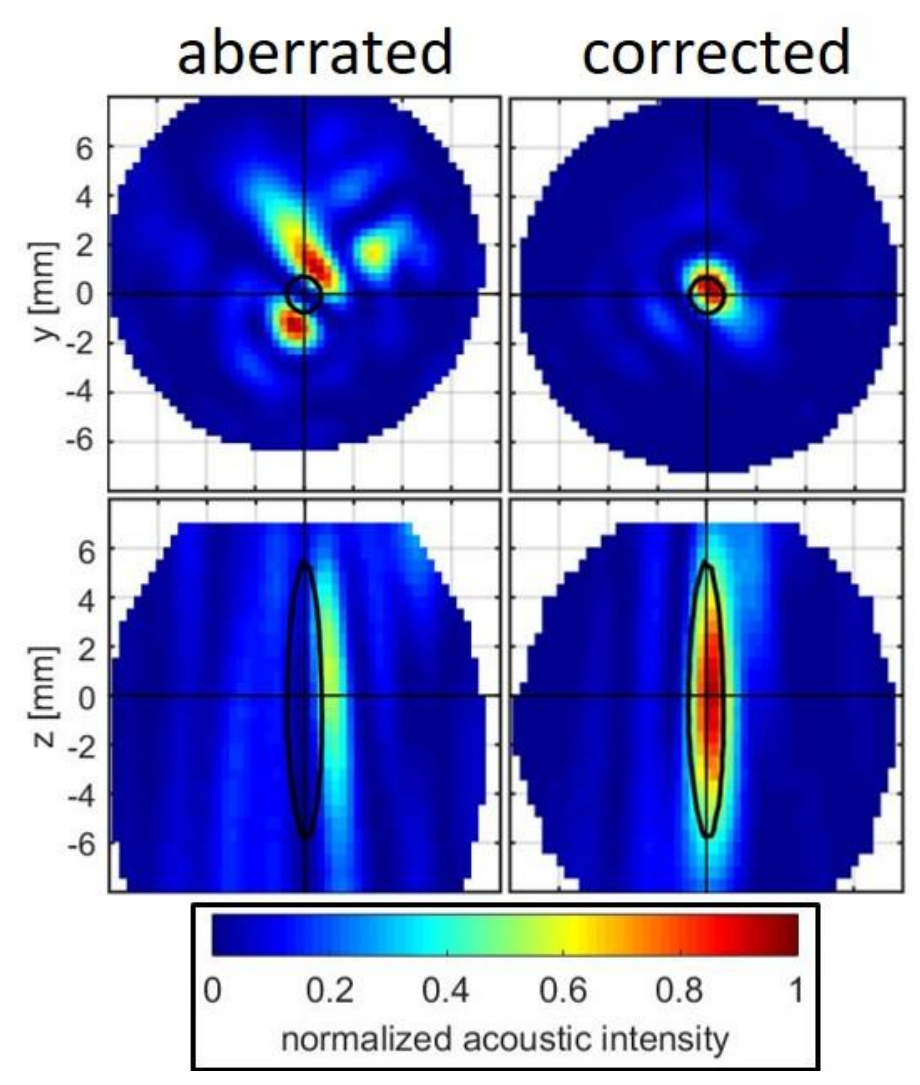

Figure 7: The acoustic intensity in the targeted area for skull A. Left: aberrated scan, right: corrected scan. For each case, an axial and a transverse view centered on the target (the acoustic focus acting as reference) are presented. The black cursor points at the target and the $-3 d B$ focus area of the reference scan is outlined in black.

\begin{tabular}{|c|c|c|c|c|c|c|}
\hline & skull A & skull B & skull C & mean \pm std & FWHM for reference scan \\
\hline \multirow{2}{*}{\begin{tabular}{|c|} 
longitudinal \\
error
\end{tabular}} & aberrated & 0.16 & 1.00 & 1.68 & $0.94 \pm 0.76$ & \multirow{2}{*}{$11 \mathrm{~mm}$} \\
\hline & corrected & 0.35 & 1.55 & 0.41 & $0.77 \pm 0.68$ & \\
\hline \multirow{2}{*}{$\begin{array}{c}\text { transverse } \\
\text { error }\end{array}$} & aberrated & 2.19 & 1.52 & 2.68 & $2.13 \pm 0.58$ & \multirow{2}{*}{$1.5 \mathrm{~mm}$} \\
\hline & corrected & 0.31 & 0.47 & 0.29 & $0.36 \pm 0.10$ & \\
\hline
\end{tabular}

Table 3: Position errors (longitudinal and transverse), before and after correction by the acoustic lens, in relation to the target defined by the reference scan. The Full Width at Half Maximum of the reference focal spot is given for comparison purposes.

\section{Focal volume and energy scattering}

Two parameters were computed to quantify the quality of the acoustic intensity focusing. The first one was the volume of the $-3 \mathrm{~dB}$ focal spot (table 4 , light green). The second one was the percentage of acoustic intensity of the aberrated $\left(I_{\text {aber }}\right)$ and lens-corrected $\left(I_{\text {cor }}\right)$ scans within the $-3 \mathrm{~dB}$ focal spot reference volume $V_{\text {ref }}$ measured in the absence of skull (Table 4, light red).

In average, while the $-3 \mathrm{~dB}$ volume increases by $471+/-231 \%$ for the aberrated scan, it only increases by $86+/-28 \%$ for the corrected scan. The acoustic lens thus limits energy scattering. Due to the defocusing effect of the skull, only $0.7+/-0.8 \%$ of the 
acoustic intensity falls within the $-3 \mathrm{~dB}$ target without any correction. With the lenscorrection $48+-2.0 \%$ of the acoustic intensity falls within the $-3 \mathrm{~dB}$ target, as calculated from Table 4 (light red).

\begin{tabular}{|c|c|c|c|c|}
\hline & & skull $A$ & skull B & skull C \\
\hline \multirow{3}{*}{$\begin{array}{c}\text { volume of the }-3 \mathrm{~dB} \text { focal spot } \\
\text { (and increase compared to } \\
\text { reference) }\end{array}$} & reference & $8.5 \mathrm{~mm}^{3}$ & $10.9 \mathrm{~mm}^{3}$ & $9.7 \mathrm{~mm}^{3}$ \\
\hline & aberrated & $69.8 \mathrm{~mm}^{3}(+721 \%)$ & $57.6 \mathrm{~mm}^{3}(+428 \%)$ & $35.4 \mathrm{~mm}^{3}(+265 \%)$ \\
\hline & corrected & $18.4 \mathrm{~mm}^{3}(+116 \%)$ & $17.4 \mathrm{~mm}^{3}(+60 \%)$ & $17.7 \mathrm{~mm}^{3}(+82 \%)$ \\
\hline \multirow{2}{*}{$\begin{array}{l}\text { percentage of the acoustic } \\
\text { intensity in the volume } V_{\text {ref }}\end{array}$} & aberrated & $1.5 \%$ & $0.5 \%$ & $0.0 \%$ \\
\hline & corrected & $50.0 \%$ & $48.9 \%$ & $46.3 \%$ \\
\hline
\end{tabular}

Table 4: Volumes of the -3dB focal spots (light green), in comparison with the reference, for the aberrated and corrected scans and percentage of the acoustic intensity found within the reference volume $V_{\text {ref }}$ (light red) for the aberrated $\left(I_{\text {aber }}\right)$ and corrected $\left(I_{\text {cor }}\right)$ scans.

\section{Acoustic Intensity}

Figure 8 shows, for skull $A$, the acoustic intensity obtained on the x-axis. The acoustic intensity at the target was also estimated (Table 5), derated by the skull and/or lens absorption as explained in the methods. The intensity obtained for the aberrated and the lens-corrected scans are compared with those obtained for the reference scan. The intensity reaches, on average, $6.0+/-1.7$ of the reference energy for the aberrated scan, whereas it reaches $59+/-22 \%$ for the corrected scan.

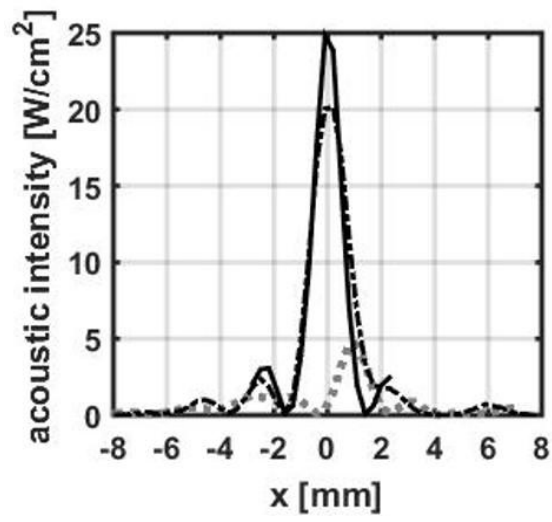

Figure 8: Acoustic intensity on the $x$-axis for skull A. Solid line: reference scan; grey dotted line: aberrated scan; dash-dot line: corrected scan. As indicated in the Methods, the intensity of the reference scan was corrected by the attenuation of the skull, and that of the corrected scan was corrected by the attenuation of the acoustic lens.

\begin{tabular}{|c|c|c|c|c|}
\hline & skull A & skull B & skull C & mean \pm std \\
\hline reference & $25 \mathrm{~W} / \mathrm{cm}^{2}$ & $17 \mathrm{~W} / \mathrm{cm}^{2}$ & $27 \mathrm{~W} / \mathrm{cm}^{2}$ & $23 \pm 5.3 \mathrm{~W} / \mathrm{cm}^{2}$ \\
\hline aberrated & $1.2 \mathrm{~W} / \mathrm{cm}^{2}(5.0 \%)$ & $1.3 \mathrm{~W} / \mathrm{cm}^{2}(7.9 \%)$ & $1.3 \mathrm{~W} / \mathrm{cm}^{2}(5.0 \%)$ & $1.3 \pm 0.1 \mathrm{~W} / \mathrm{cm}^{2}(6.0 \pm 1.7 \%)$ \\
\hline corrected & $18 \mathrm{~W} / \mathrm{cm}^{2}(72 \%)$ & $12 \mathrm{~W} / \mathrm{cm}^{2}(71 \%)$ & $9.2 \mathrm{~W} / \mathrm{cm}^{2}(34 \%)$ & $13 \pm 4.5 \mathrm{~W} / \mathrm{cm}^{2}(59 \pm 22 \%)$ \\
\hline
\end{tabular}

Table 5: Acoustic intensity obtained at the target point $(x=0, y=0, z=0)$. 


\section{Discussion}

We demonstrated experimentally that the transcranial focusing quality of a singleelement transducer can be significantly improved by using a skull-specific corrective acoustic lens, as shown on three different human skulls. The position error of the acoustic focus (Table 3 ) compared to the target location equals $24 \%$ of the reference scan FWHM transversally and $7.0 \%$ axially. The intensity at the target location is on average 10 times higher with the lens (Table 5). This technique thus ensures a high quality focusing (precise and with fewer undesirable side lobe effects), making it a promising candidate for applications in low-power transcranial therapies such as BBB opening and neurostimulation/neuromodulation.

To improve the quality of the focusing even more and reduce side lobe levels, an inverse filter approach $[55,56]$ could be used to determine the lens shape instead of the time-shift method used in our study. Using compound materials in the making of the lens can also be considered to modulate the amplitude of the wave in addition to its phase.

In this study, the impact of refraction and diffraction at the boundaries of the lens were not taken into account when computing its thickness. Numerical simulations demonstrated that omitting these phenomena did not keep the lens-based aberration correction method from outperforming that of a multi-element transducer featuring $6 \times 6 \mathrm{~mm}^{2}$ elements: the lens-based method concentrates $8 \%$ more acoustic intensity at the focus. However, the acoustic intensity obtained with the acoustic lens remains $24 \%$ lower than what could be achieved with a theoretical gold standard (a transducer featuring elements that are the size of the spatial step of the simulation). These results indicate that the quality of the focusing obtained using the acoustic lens could further be improved by taking into account the aforementioned effects.

As far as the acoustic intensity is concerned, the acoustic lens allowed us to reach $59 \%$ of the optimal level (reference scan) that we could expect on the acoustic target (Table 5). Comparatively, two studies conducted with multi-element arrays showed similar correction effectiveness. Clement et al. showed that a method based on a layered wavevector-frequency domain model allowed to reach $45 \%$ of the optimal energy at $740 \mathrm{kHz}$ [15]. Marsac et al. obtained $74 \%$ of the optimal energy at $1 \mathrm{MHz}$ with a method based on a 3D-CT scan and a finite difference time domain simulation [49].

The transducer used here was sufficient to perform a proof of concept. Nevertheless, from a practical standpoint, a transducer with a larger active surface, akin to these currently in use in transcranial therapy by multi-element arrays, should be preferred. Indeed, current arrays are typically much larger, with the following specifications: $15 \mathrm{~cm}$ focus and $23 \mathrm{~cm}$ aperture [21] for the one developed by the Institut Langevin (Paris, France); $15 \mathrm{~cm}$ focus and $30 \mathrm{~cm}$ aperture [33, 57] for the ExAblate 4000 developed by Insightec (Tirat Carmel, Israel). A larger surface allow for an increase 
in antenna gain and could significantly increase the Specific Absorption Rate (SAR) at the focus compared to that measured at the surface of the skull. In that case, it could then become feasible to carry out thermal necrosis with this method.

On average, the acoustic attenuation of the skull was found to be equal to $12.0 \pm 1.0 \mathrm{~dB}$ at $914 \mathrm{kHz}$. Pinton et al. reported a $13.3 \mathrm{~dB} / \mathrm{cm}$ absorption for a human skull at $1 \mathrm{MHz}$ [3], which is in line with our measurement, taking into account an average $7.0 \mathrm{~mm}$ and $6.7 \mathrm{~mm}$ thickness of male and female human skulls respectively [58].

This method allows for a high-quality correction of the aberrations induced by the propagation through a skull, even for frequencies around $1 \mathrm{MHz}$, which are known to be particularly challenging for current multi-element-based methods $[4,59]$. The characteristic length of the thickness variation for the lens is below one millimeter while the resolution of the 3D printer used here is around $300 \mu \mathrm{m}$ in the $\mathrm{XY}$ directions. The transducer used in our study (whose radius of curvature is $59 \mathrm{~mm}$ and f-number is 1) can thus be assumed to be roughly equivalent to a 3000 -element array, which could explain why an acoustic-lens-based method still performs well at higher frequencies. While teams throughout the world are racing to build arrays with an ever growing number of elements, the acoustic lens approach could appear as a promising disruptive technology by allowing submillimetric phase correction over a large surface with a limited cost.

In comparison with multi-element arrays, using a single element is a compact size, easy-to-use and affordable solution. Its compactness would allow an easier integration in MR environments which are adapted for real-time monitoring of treatments. Furthermore, using a single element considerably reduces costs. It is also worth noting that lens design and production could be performed off-site, using uploaded CT-images, and then shipped to the treatment site. Such an approach would further reduce the amount of equipment and skills required on site, at each therapeutic center.

This technique is both patient-specific and target-specific, meaning that a new lens has to be made for each case. However, a given lens could be used repeatedly for the purposes of treating chronic diseases by repeatedly targeting the same area. This approach suits particularly well neurologic disorders for which small volumes would be targeted (subgenual anterior cingulate cortex (sgACC) in depression, deep thalamic nuclei in dystonia).

One of the disadvantages lies in the optimization of the material's properties, in order to limit thermal rise in the acoustic lens. Indeed, the modification of the speed of sound inside the lens, due to acoustic heating, could potentially act as a thermal lens $[60,61]$ during long, lasting sequences of high intensity focused ultrasound, and these effects should be studied, and minimized if needed. 
The steering capabilities of the lens could also be questioned. Previous studies proved that steering by simply tilting the wave front was effective for low isoplanetism angles $[8,62]$. Pernot et al. used such tilting to induce thermal necrosis on a $30 \times 30 \times 40 \mathrm{~mm}^{3}$ volume around the focal region [38], based on a skull aberration correction calculated at the center of the volume. The ability to provide adequate mechanical steering possibilities to the transducer, covered with the lens apparatus, is beyond the scope of this paper and will be investigated in further studies.

Lastly, the method used here to position the transducer - a 3D-printed holder maintained with rubber bands - gave a satisfactory level of precision. Translation deviations were estimated to $1 \mathrm{~mm}$, and rotation deviations to less than 5 degrees. As evidenced throughout our experiments, such errors were not detrimental to the accuracy of the correction and targeting. Complementary experiments are required to quantitatively figure out the effectiveness of this approach. Clinical transfers could require thermoplastic masks, like the ones used in radiosurgery [63,64], to ensure precise, in vivo positioning of the transducer and further simplify the targeting procedure. Neuronavigation could also facilitate the positioning.

\section{Conclusion}

The objective of this study was to experimentally demonstrate that correcting skullinduced aberrations, using a single-element transducer combined with a specific acoustic lens, is achievable. To our knowledge, this is the first time that this technology is used in the context of ultrasonic transcranial focusing for aberration correction. While the principle of acoustic lenses has been known for a long time, its practical application to this specific case requires complete control of the production process from numerical modeling, simulation, to 3D printing and positioning, which technological breakthroughs have made possible only very recently.

Our experiments on a sample of three human skulls demonstrated the effectiveness of this method. On account of the simplicity of its implementation and affordable price, we consider that this method holds genuine clinical potential for ultrasonic brain therapy.

\section{Acknowledgements}

This work was supported by the Bettencourt Schueller Foundation and the "Agence Nationale de la Recherche" under the program "Future Investments" with the reference ANR-10-EQPX-15.

1. Fry, W.J., et al., Production of focal destructive lesions in the central nervous system with ultrasound. Journal of Neurosurgery, 1954: p. 471-479.

2. Barnard, J.W., et al., Effects of high intensity ultrasound on the central nervous system of the cat. Journal of Comparative Neurology, 1955. 103(3): p. 459-484.

3. Pinton, G., et al., Attenuation, scattering, and absorption of ultrasound in the skull bone. Medical physics, 2012. 39(1): p. 299-307. 
4. Fry, F.J. and J.E. Barger, Acoustical properties of the human skull. The Journal of the Acoustical Society of America, 1978. 63(5): p. 1576-1590.

5. Misra, A., et al., Drug delivery to the central nervous system: a review. J Pharm Pharm Sci, 2003. 6(2): p. 252-73.

6. Elias, W.J., et al., A magnetic resonance imaging, histological, and dose modeling comparison of focused ultrasound, radiofrequency, and Gamma Knife radiosurgery lesions in swine thalamus. Journal of Neurosurgery, 2013. 119(2): p. 307-317.

7. Hynynen, K. and N. McDannold, MRI guided and monitored focused ultrasound thermal ablation methods: a review of progress. International journal of hyperthermia, 2004. 20(7): p. 725-737.

8. Tanter, M., J.-L. Thomas, and M. Fink, Focusing and steering through absorbing and aberrating layers: application to ultrasonic propagation through the skull. The Journal of the Acoustical Society of America, 1998. 103(5): p. 2403-2410.

9. Tanter, M., J.-L. Thomas, and M. Fink. Focusing through skull with time reversal mirrors. Application to hyperthermia. in Ultrasonics Symposium, 1996. Proceedings., 1996 IEEE. 1996. IEEE.

10. Sun, J. and K. Hynynen, Focusing of therapeutic ultrasound through a human skull: a numerical study. J Acoust Soc Am, 1998. 104(3 Pt 1): p. 1705-15.

11. Hynynen, K. and F.A. Jolesz, Demonstration of potential noninvasive ultrasound brain therapy through an intact skull. Ultrasound Med Biol, 1998. 24(2): p. 275-83.

12. Thomas, J.-L. and M.A. Fink, Ultrasonic beam focusing through tissue inhomogeneities with a time reversal mirror: application to transskull therapy. IEEE transactions on ultrasonics, ferroelectrics, and frequency control, 1996. 43(6): p. 1122-1129.

13. Aubry, J.F., et al., Experimental demonstration of noninvasive transskull adaptive focusing based on prior computed tomography scans. J Acoust Soc Am, 2003. 113(1): p. 84-93.

14. Marquet, F., et al., Non-invasive transcranial ultrasound therapy guided by CT-scans. Conf Proc IEEE Eng Med Biol Soc, 2006. 1: p. 683-7.

15. Clement, G. and K. Hynynen, A non-invasive method for focusing ultrasound through the human skull. Physics in Medicine and Biology, 2002. 47: p. 1219-1236.

16. Cohen, Z.R., et al., Magnetic resonance imaging-guided focused ultrasound for thermal ablation in the brain: a feasibility study in a swine model. Neurosurgery, 2007. 60(4): p. 593600; discussion 600.

17. Pernot, M., et al., In vivo transcranial brain surgery with an ultrasonic time reversal mirror. J Neurosurg, 2007. 106(6): p. 1061-6.

18. Hynynen, K., et al., Pre-clinical testing of a phased array ultrasound system for MRI-guided noninvasive surgery of the brain-a primate study. European journal of radiology, 2006. 59(2): p. 149-156.

19. Chauvet, D., et al., Targeting accuracy of transcranial magnetic resonance-guided highintensity focused ultrasound brain therapy: a fresh cadaver model. Journal of Neurosurgery, 2013. 118(5): p. 1046-1052.

20. Eames, M.D., et al., Trans-cranial focused ultrasound without hair shaving: feasibility study in an ex vivo cadaver model. Journal of Therapeutic Ultrasound, 2014. 1(1): p. 1-6.

21. Marsac, L., et al., MR-guided adaptive focusing of therapeutic ultrasound beams in the human head. Medical Physics, 2012. 39: p. 1141.

22. Marquet, F., et al., Real-time, transcranial monitoring of safe blood-brain barrier opening in non-human primates. PloS one, 2014. 9(2): p. e84310.

23. McDannold, N., et al., Temporary disruption of the blood-brain barrier by use of ultrasound and microbubbles: safety and efficacy evaluation in rhesus macaques. Cancer research, 2012. 72(14): p. 3652-3663.

24. Hynynen, K., et al., Noninvasive MR imaging-guided focal opening of the blood-brain barrier in rabbits. Radiology, 2001. 220(3): p. 640-6. 
25. Liu, H.L., et al., Hemorrhage detection during focused-ultrasound induced blood-brain-barrier opening by using susceptibility-weighted magnetic resonance imaging. Ultrasound Med Biol, 2008. 34(4): p. 598-606.

26. Marquet, F., et al., Non-invasive ultrasonic surgery of the brain in non-human primates. The Journal of the Acoustical Society of America, 2013. 134(2): p. 1632-1639.

27. Deffieux, T., et al., Low-intensity focused ultrasound modulates monkey visuomotor behavior. Current Biology, 2013. 23(23): p. 2430-2433.

28. King, R.L., et al., Effective Parameters for Ultrasound-Induced In Vivo Neurostimulation. Ultrasound in Medicine \& Biology, 2013. 39(2): p. 312-331.

29. Tufail, Y., et al., Ultrasonic neuromodulation by brain stimulation with transcranial ultrasound. Nat. Protocols, 2011. 6(9): p. 1453-1470.

30. Yoo, S.-S., et al., Focused ultrasound modulates region-specific brain activity. Neurolmage, 2011. 56(3): p. 1267-1275.

31. Younan, Y., et al., Influence of the pressure field distribution in transcranial ultrasonic neurostimulation. Medical physics, 2013. 40(8): p. 082902.

32. Legon, W., et al., Transcranial focused ultrasound modulates the activity of primary somatosensory cortex in humans. Nature neuroscience, 2014. 17(2): p. 322-329.

33. Martin, E., et al., High-intensity focused ultrasound for noninvasive functional neurosurgery. Ann Neurol, 2009. 66(6): p. 858-61.

34. Elias, W.J., et al., A Pilot Study of Focused Ultrasound Thalamotomy for Essential Tremor. New England Journal of Medicine, 2013. 369(7): p. 640-648.

35. Lipsman, N., et al., MR-guided focused ultrasound thalamotomy for essential tremor: $a$ proof-of-concept study. The Lancet Neurology, 2013. 12(5): p. 462-468.

36. Chang, W.S., et al., Unilateral magnetic resonance guided focused ultrasound thalamotomy for essential tremor: practices and clinicoradiological outcomes. Journal of Neurology, Neurosurgery \& Psychiatry, 2014: p. jnnp-2014-307642.

37. Clement, G.T., et al., A hemisphere array for non-invasive ultrasound brain therapy and surgery. Phys Med Biol, 2000. 45(12): p. 3707-19.

38. Pernot, M., et al., High power transcranial beam steering for ultrasonic brain therapy. Phys Med Biol, 2003. 48(16): p. 2577-89.

39. Jeanmonod, D., et al., Transcranial magnetic resonance imaging-guided focused ultrasound: noninvasive central lateral thalamotomy for chronic neuropathic pain. Neurosurgical Focus, 2012. 32(1): p. 1-11.

40. Lele, P., An annular-focus ultrasonic lens for production of uniform hyperthermia in cancer therapy. Ultrasound in Medicine \& Biology, 1981. 7(2): p. 191-193.

41. Umemura, S., C. Cain, and K. Katakura. Acoustic field synthesis by sector-vortex array for tumor treatment. in IEEE 1986 Ultrasonics Symposium. 1986. IEEE.

42. Cain, C.A. and S.-I. Umemura, Concentric-ring and sector-vortex phased-array applicators for ultrasound hyperthermia. IEEE Transactions on Microwave Theory and Techniques, 1986. 34(5): p. 542-551.

43. Lalonde, R., A. Worthington, and J. Hunt. Hyperthermia: Field conjugate acoustic lenses for deep heating. in Engineering in Medicine and Biology Society, 1990., Proceedings of the Twelfth Annual International Conference of the IEEE. 1990. IEEE.

44. Lalonde, R. and J.W. Hunt, Variable frequency field conjugate lenses for ultrasound hyperthermia. IEEE transactions on ultrasonics, ferroelectrics, and frequency control, 1995. 42(5): p. 825-831.

45. Takayama, N. and T. Itoh. Investigation of ultrasonic heating with a non-axial symmetric acoustic lens. in Proc. 5th Int. Symp. Hyperthermic Oncology. 1989.

46. Fjield, T. and K. Hynynen, The combined concentric-ring and sector-vortex phased array for $M R I$ guided ultrasound surgery. IEEE transactions on ultrasonics, ferroelectrics, and frequency control, 1997. 44(5): p. 1157-1167. 
47. Brown, M., et al., Generating arbitrary ultrasound fields with tailored optoacoustic surface profiles. Applied Physics Letters, 2017. 110(9): p. 094102.

48. Fjield, T., C.E. Silcox, and K. Hynynen, Low-profile lenses for ultrasound surgery. Phys Med Biol, 1999. 44(7): p. 1803-13.

49. L. Marsac, D.C., R. La Greca, A-L Boch, K. Chaumoitre, M. Tanter, J-F Aubry, Ex vivo optimization of a heterogeneous speed of sound model of the human skull for non-invasive transcranial focused ultrasound at $1 \mathrm{MHz}$. International journal of hyperthermia, 2017.

50. Marsac, L., et al., Ex vivo optimisation of a heterogeneous speed of sound model of the human skull for non-invasive transcranial focused ultrasound at $1 \mathrm{MHz}$. International Journal of Hyperthermia, 2017: p. 1-11.

51. Duck, F.A., Physical properties of tissues: a comprehensive reference book. 1990: Academic Press.

52. Cox, B.T., et al., k-space propagation models for acoustically heterogeneous media: Application to biomedical photoacoustics. The Journal of the Acoustical Society of America, 2007. 121(6): p. 3453-3464.

53. Ghiglia, D.C. and M.D. Pritt, Two-dimensional phase unwrapping: theory, algorithms, and software. Vol. 4. 1998: Wiley New York.

54. TiePie. Handyscope HS5 downloads. 2017; Available from: https://www.tiepie.com/en/products/Oscilloscopes/Handyscope HS5/Download.

55. Tanter, M., et al., Optimal focusing by spatio-temporal inverse filter. I. Basic principles. The Journal of the Acoustical Society of America, 2001. 110(1): p. 37-47.

56. Aubry, J.-F., et al., Optimal focusing by spatio-temporal inverse filter. II. Experiments. Application to focusing through absorbing and reverberating media. The Journal of the Acoustical Society of America, 2001. 110(1): p. 48-58.

57. Arvanitis, C.D., M.S. Livingstone, and N. McDannold, Combined ultrasound and MR imaging to guide focused ultrasound therapies in the brain. Physics in medicine and biology, 2013. 58(14): p. 4749.

58. Lynnerup, N., Cranial thickness in relation to age, sex and general body build in a Danish forensic sample. Forensic science international, 2001. 117(1): p. 45-51.

59. Fry, F.J., Transkull transmission of an intense focused ultrasonic beam. Ultrasound Med Biol, 1977. 3(2-3): p. 179-84.

60. Hallaj, I.M., R.O. Cleveland, and K. Hynynen, Simulations of the thermo-acoustic lens effect during focused ultrasound surgery. J Acoust Soc Am, 2001. 109(5 Pt 1): p. 2245-53.

61. Floch, C.L., M. Tanter, and M. Fink, Self-defocusing in ultrasonic hyperthermia: Experiment and simulation. Applied physics letters, 1999. 74(20): p. 3062-3064.

62. Clement, G.T. and K. Hynynen, Micro-receiver guided transcranial beam steering. Ultrasonics, Ferroelectrics and Frequency Control, IEEE Transactions on, 2002. 49(4): p. 447453.

63. Minniti, G., et al., Frameless linac-based stereotactic radiosurgery (SRS) for brain metastases: analysis of patient repositioning using a mask fixation system and clinical outcomes. Radiation Oncology, 2011. 6(1): p. 158.

64. van Santvoort, J., R. Wiggenraad, and P. Bos, Positioning accuracy in stereotactic radiotherapy using a mask system with added vacuum mouth piece and stereoscopic $X$-ray positioning. International Journal of Radiation Oncology* Biology* Physics, 2008. 72(1): p. 261-267. 